\title{
REVIEW ON TIME-FREQUENCY-BASED MACHINE LEARNING FOR STRUCTURAL DAMAGE ASSESSMENT AND CONDITION MONITORING
}

\author{
Rims Janeliukstis \\ Riga Technical University, Latvia \\ rims.janeliukstis_1@rtu.lv
}

\begin{abstract}
Safe operation of complex structures and machines cannot be achieved by visual inspections alone. Thus, simple, efficient and cost-effective inspection methods are of paramount importance. This review article is aimed at providing an insight into approaches of health monitoring and condition monitoring of structures and machines based on machine learning. Machine learning is an approach of using data to construct predictive models giving predictions of existing and future trends. In the case of structural health monitoring and condition monitoring of machinery, it is able to detect damage and distinguish between different damage types and severities, thus being able to provide early warnings of failure of structural and machinery components. In this review paper, the focus is on information obtained from time-frequency features, which provides an advantage with respect to features in time domain and frequency domain of tracking changes of structural integrity in time, which is especially useful in dealing with non-stationary signals. Details of damage-sensitive feature extraction, data analysis and decision making are provided.
\end{abstract}

Keywords: time-frequency, monitoring, damage, machine learning.

\section{Introduction}

The ever increasing needs for safe and reliable infrastructure, transportation and machinery have facilitated a rapid development of structural health monitoring (SHM) and condition monitoring (CM) domains. The interrogation of integrity of structures and machines is a multidisciplinary field spanning such branches of engineering as civil, mechanical and aeronautical engineering. The advancement of these techniques offers a tremendous asset to maintaining and preserving the city infrastructure, various machinery as well as engineering communication networks, which is crucial for units operating in harsh environmental conditions. Moreover, these methods help in reducing structural and machinery inspection costs and unwanted operational downtime with an added reliability. For example, in the case of wind turbine generators, about $25 \%$ to $30 \%$ of the overall wind power generation costs account for operation and maintenance, while wind turbine blade failure results in more than 7 days of downtime [1]. The approach of SHM and CM exploits collection and analysis of signals continuously measured with mounted sensors or visual images captured with aerial vehicles [1] or laser scanners [2].

The most popular branch of methods for structural and machinery diagnostics is based on vibration monitoring, because vibration signals can be measured with relative ease and they correlate well with the working conditions of rotating machinery [3]. By extracting relative damage-sensitive features from the measured vibration signals (either displacement, velocity or acceleration) in time domain of frequency domain and applying pattern recognition methods like supervised or unsupervised learning schemes, the developed algorithms are capable of detecting and possibly localizing the damage. In some cases, estimation of damage severity is also possible.

The advantage of machine learning in $\mathrm{CM}$ of rotating machinery with respect to the traditional $\mathrm{CM}$ approach is that traditional approaches are not suitable in case of non-stationary signals, when frequency components change over time due to wear and tear of rotating components [4]. Machine learning provides means to build damage monitoring algorithms that learn from the data and adapt to the current situation [4].

The effectiveness of the steps mentioned above is enhanced by considering a time-frequency domain, because many real processes are not infinitely periodic (which is the case with the Fourier Transform), but rather contain some transient components. Hence, these components are localized in time and cannot be grasped by traditional spectral analysis. The most popular technique for timefrequency analysis is wavelet transform, which can be used for both stationary and non-stationary signals [3]. This method can also be effectively applied to structural damage interrogation composite structures to track delamination damage [5;6] and concrete structures to monitor crack propagation [2] among other cases. 


\section{Time-frequency transform}

The most popular time-frequency transform techniques are wavelet transform and the HilbertHuang transform. This paper will focus on the wavelet transform. Wavelets $\psi(t)$ are special functions with small oscillations and zero mean. Mother wavelet functions can be translated (parameter $a$ ) and dilated (scale parameter $s$ ) over the axis to obtain daughter wavelet functions $\psi_{a, s}(t)$. Continuous wavelet transform (CWT) is a convolution of daughter wavelet with a signal $x(t)$ and essentially measures a correlation between the two giving rise to the wavelet transform coefficients $W_{x}(a, s)$ :

$$
W_{x}(a, s)=\int_{-\infty}^{\infty} x(t) \frac{1}{\sqrt{|s|}} \psi^{*}{ }_{a, s}\left(\frac{t-a}{s}\right) d t
$$

The coefficients of CWT can be viewedas a 3-D plot in the time-frequency domain, where the amplitude of the wavelet transform coefficients is perpendicular to the plane of time-frequency (wavelet scale). A discrete version of wavelet transform, called discrete wavelet transform (DWT) is also used. It is particularly useful to avoid the redundancy of CWT where transform coefficients are computed at every scale. The most important strength of wavelet transform in fault detection is the adaptability of time-frequency resolution - high time resolution, but low frequency resolution is attained at high frequencies and vice versa is true at low frequencies [3].

\section{Damage-sensitive features}

Statistical features of the wavelet coefficient plot obtained from CWTcan be extracted and used as an input for a pattern recognition scheme. The commonly used statistical features (sequentially expressed with $W_{1}: W_{K}$, where $K$ stands for the number of features) among others are [4;7]: mean value, standard deviation, variance, RMS (root-mean-square), absolute maximum, kewness, kurtosis, crest factor, total energy of the signal.

\section{Fault discrimination strategy}

A typical experimental setup for structural damage detection or condition monitoring using machine learning is shown in Fig.1 (a). Usually, piezoelectric accelerometer sensors are used to measure vibrations in form of acceleration in the case of rotating machines (gearbox) as accelerometers are easy to mount, they have low noise output, they have a wide frequency range and dynamic range and do not have any moving parts meaning the measurements are reliable [8]. Nowadays, modern wireless microelectromechanical (MEMS) accelerometers provide additional advantages of small size and mass with no extensive electrical cabling, which is perfectly suited for fault monitoring of light and sensitive machines and structures [9]. Tachometer is used to measure the rotation speed of a shaft. Delamination interrogation in wind turbine blades is shown in Fig.1 (b). Transmitter and receiver transducers are required. The force wave emitted by the transmitter is propagating through the structure and this signal is sensed by the receiver. The contents of the received signal (amplitude, frequency) are modified due to damage.

Machine learning is a valuable tool in retrieving valuable information from the data. In order for the machine learning approach to be effective in damage detection and monitoring, a reference set of values (signal features from healthy structure/machine) have to be collected so that they are compared to the corresponding feature sets of the damaged component.

Features of signals from healthy and damaged components can be either effectively grouped together in clusters, when no information is available on the nature of these signals (unsupervised learning), or they can be classified into several categories based on class labels. For example, "healthy", "light damage", "severe damage" or "damage type 1", "damage type 2", etc. This later mode of grouping is called supervised learning. A common type of supervised machine learning is a classification where features are assigned a class. Various classification algorithms exist, among which the most popular are decision trees, discriminant analysis, $k$-nearest neighbours, naïve Bayes, support vector machines and artificial neural networks among others [11]. Artificial neural networks (ANN) are widely used in machinery fault diagnosis. The two most common variations of ANN are radial basis functions (RBF) and multi-layer perceptrons [10]. One of the most frequently used unsupervised schemes is $k$-means clustering, where data are grouped into clusters according to similarity (distance 
between data points) [12]. Smaller distance implies larger similarity between two data points and thus they belong to the same cluster. The dimensionality of data can be reduced and separability of clusters or classes can be explored using the principal component analysis (PCA).
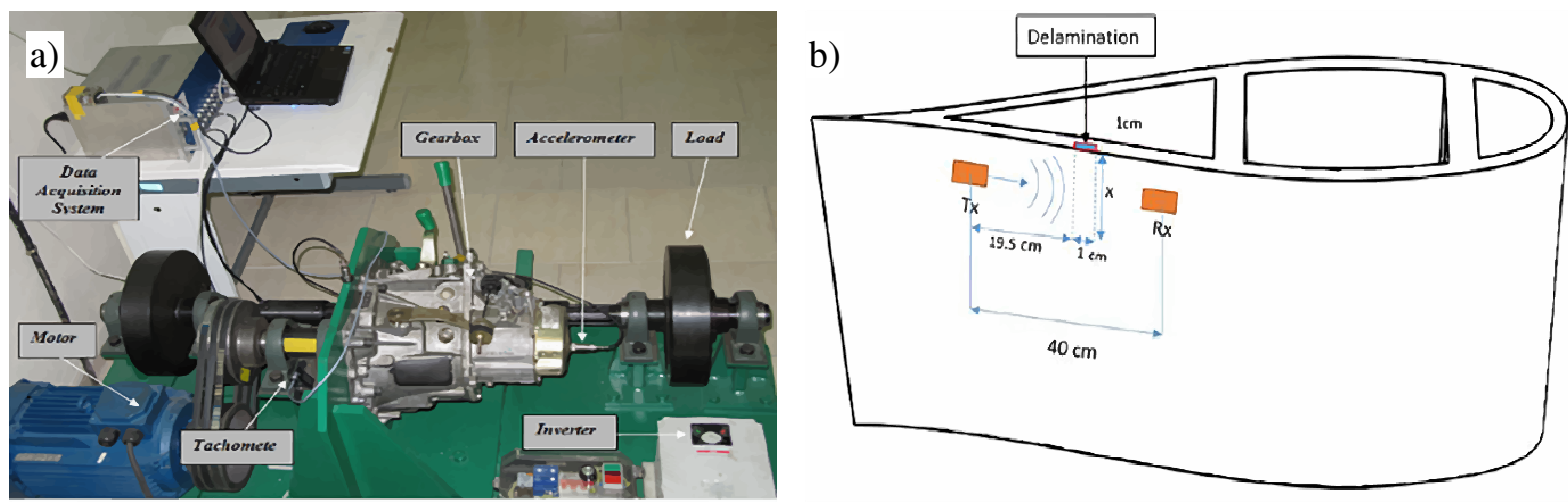

Fig. 1.Test setup for damage detection: a - gearbox condition monitoring using accelerometer as a sensor [10]; $b$ - delamination detection in wind turbine blades using concept of guided waves [5]

The strategy of fault discrimination using machine learning with time-frequency features is depicted in Fig. 2 and can be summarized into the following steps:

1. Measurement of vibration signal or some other representative signal, for example, strain [12] in time domain (Fig.2 (a) and (b)). These measurements have to be performed for the reference object at healthy state and damaged state with, possibly, several damage scenarios (different damage types or severities).

2. Wavelet transform (either discrete or continuous) of the acquired time domain signals is computed (Fig.2 (c) and (d)). One of the issue here is to select the appropriate wavelet function. In the case for rotating machinery, many researchers have used the Daubechies wavelets $\mathrm{db} 2, \mathrm{db} 4, \mathrm{db} 5, \mathrm{db} 10$ and $\mathrm{db} 40$ as a mother wavelet function and 3, 4, 8 and 10 level of decomposition of vibration signal for identification of fault in gear and bearing [7]. On the other hand, the Morlet wavelet is also used due to the fact that its shape resembles an impulse signal produced by many mechanical and dynamic systems [10]. Mexican Hat wavelet is also seldom used [2].

3. Wavelet transform coefficients are examined at different wavelet scales. Selection of scale values for damage-sensitive feature extraction depends on the problem at hand. In [7] the authors extracted the wavelet transform coefficients at scales corresponding to gear mesh frequency to detect compound faults in gears and bearings. The issue of selection of optimal scale parameters for the wavelet functions is described in [10], where wavelet entropy and energy are used to select the optimal scales of the wavelet function. The proper scales are chosen, when the Shannon entropy of the corresponding wavelet coefficients is minimum and the energy is maximum.

Wavelet Shannon entropy that measures uncertainty of wavelet coefficients is defined as

$$
H(n)=-\sum_{i=1}^{m} P_{i} \ln P_{i}
$$

$$
\text { where } P_{i}=\frac{|z(n)|^{2}}{E D(n)}-\text { distribution of the energy probability of each wavelet coefficient; }
$$

The ratio of the wavelet transform coefficient energy to the Shannon entropy is defined as

$$
\mu(n)=\frac{E D(n)}{H(n)}
$$

The rationale behind this idea is that the Shannon entropy measures similarity between energy probability distributions of wavelet transform coefficients. Hence, energy probability distributions of wavelet transform coefficients of a signal from healthy element and faulty element would be different and these changes could be tracked. 
1. Time-frequency features are extracted at these chosen wavelet scales and are then used to build a classifier. Examples of decision trees and ANN classifiers are shown in Fig.2(f) and (g), respectively. These classifiers, in essence, categorize or separate the input data into several categories based on the values of the input features. Hence, different types of damage can be distinguished.

2. In order to optimize the classifier, the various hyper-parameters of classifiers have to be finetuned and the classification model has to be cross-validated to avoid overfitting [13]. Classification performance is assessed through metrics, such as classification accuracy which shows a fraction of correct classifications.

Classes: healthy, damage type 1 , damage type $2, \ldots$, damage type $n$.

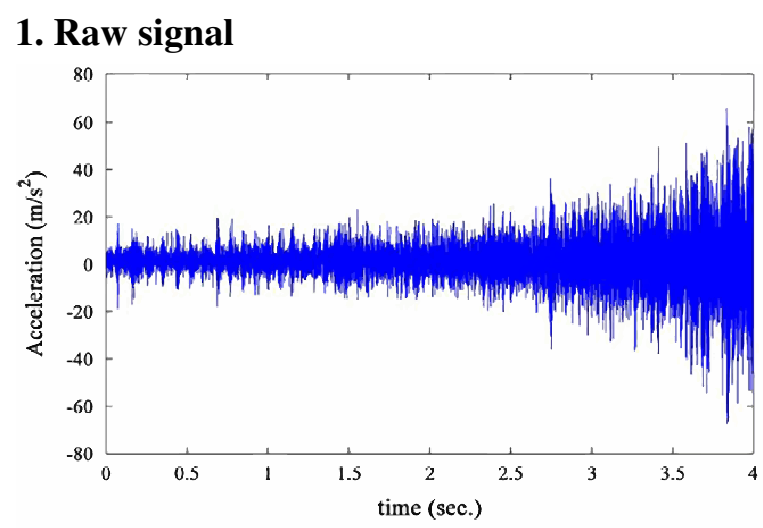

\section{Wavelet scalogram}

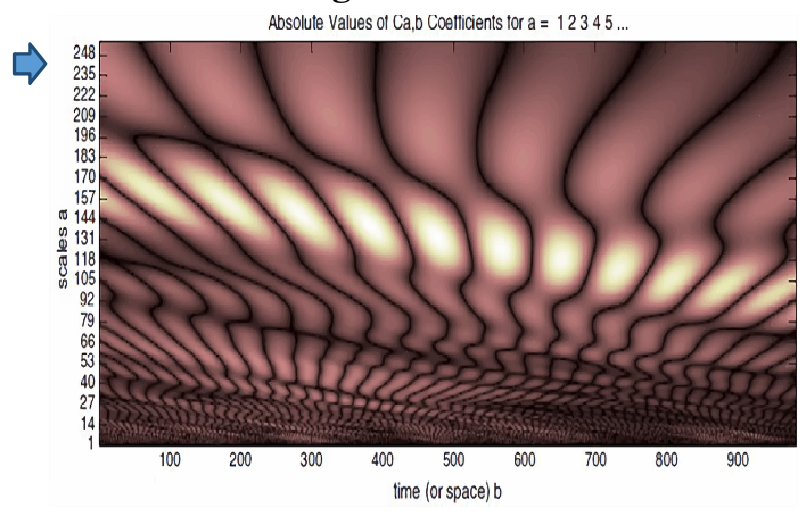

ת

3. Optimum selection of wavelet scales (levels for DWT)

4. Extract damage-sensitive features $W_{1}: W_{K}$ in time-frequency domain

\section{Build a classifier}

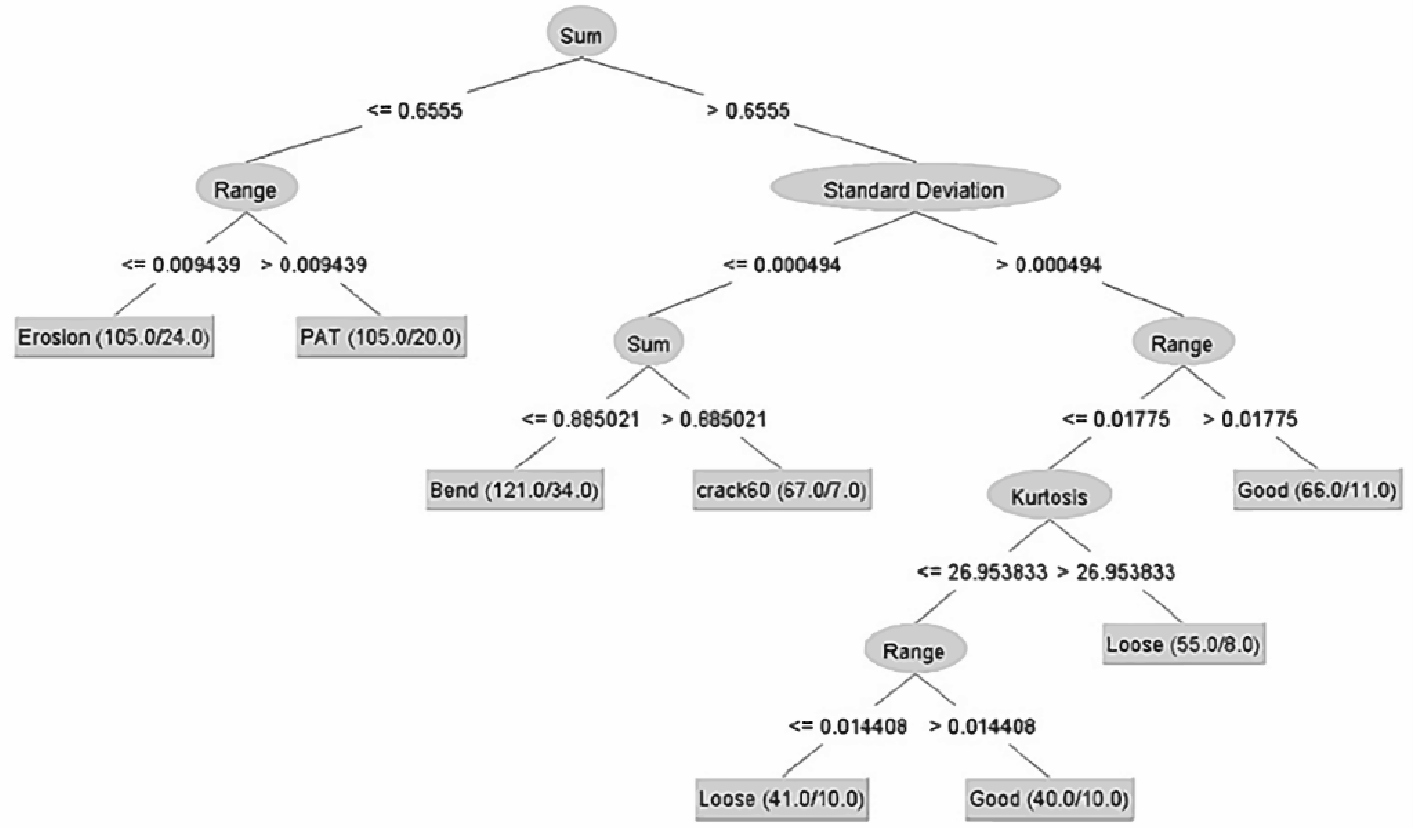

\section{Assess the condition of the structural part}

Fig. 2. Steps of fault discrimination: 1 - vibration signals of healthy and damaged elements [10];

2 - CWT coefficient plots for healthy and faulty elements [7]; 3 - optimum selection of wavelet scales; 4 - extracted features in time-frequency domain; 5 - actual classification of faults with some machine learning model, for example, decision trees [4]; 6- decision on structural safety based on the machine learning model 


\section{Advantage of time-frequency domain}

The authors in [7] compared the fault diagnostic potential of extracted damage-sensitive features from the time, frequency and time-frequency domains in order to detect a compound gear and bearing fault. They applied neural network classification algorithm and concluded that standard deviation, variance and absolute maximum values extracted from the wavelet transform coefficient plot at scale parameter values equal to the gear mesh frequency and its harmonics showed superior diagnostics potential as displayed in Fig. 3.

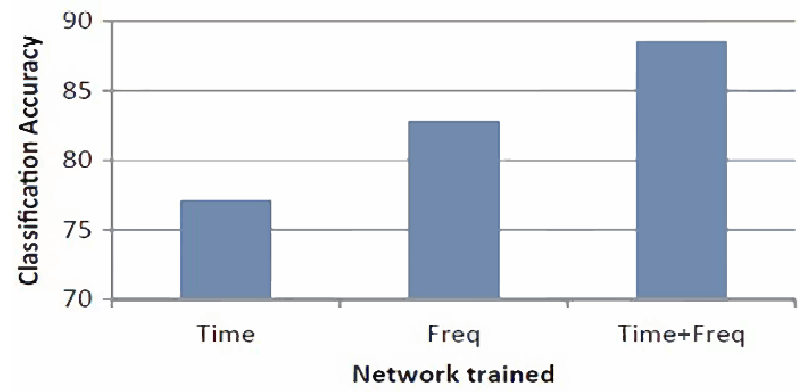

Fig. 3. Classification accuracy of neural network classifier using damage-sensitive features from time, frequency and time-frequency domains [7]

The results of PCA for distinguishing different types of damage (holes) are shown in Fig.4. As it can be seen, signal treatment with wavelet transform and relevant feature extraction (energy distribution of wavelet coefficients) improves the feature discrimination results (separability of classes) of the case when time domain features are used.

a)

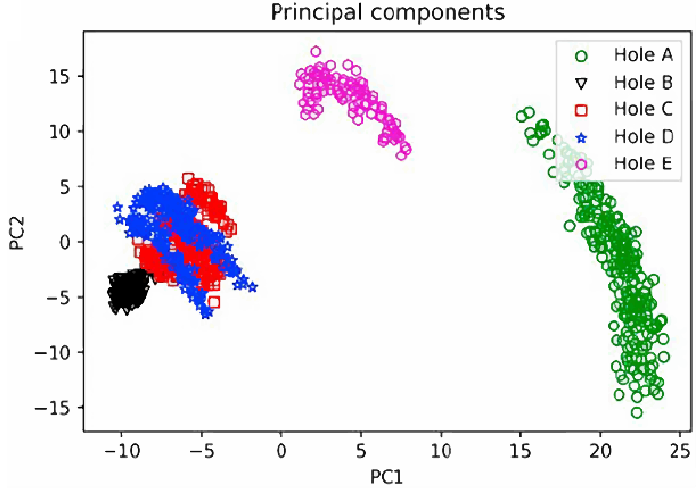

b)

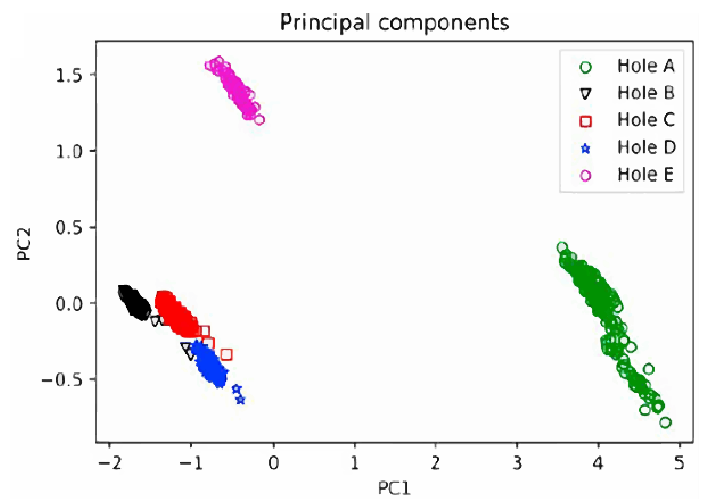

Fig. 4. Cluster separability using first two principal components distinguishing between different types of holes [12]: $a$ - original time signal is used; $b$ - wavelet transform coefficient energy distributions are used as a feature

Machine learning approach with extraction of relevant damage-sensitive features allows an effective prediction of structural damage - be it in the form of binary classification in a sense of distinguishing between healthy and damaged structure (damage detection) or a multiclass problem with either localizing defect in a pre-defined zones of the structure or discerning between different types or severities of damage.

\section{Conclusions}

The practical challenges associated with this concept are in a fact that usually massive amounts of data are needed for successful damage interrogation. Moreover, appropriate machine learning algorithms have to be selected and hyperparameters fine-tuned in order to mitigate the harmful effects of noise and outliers. Sophisticated internal regularization mechanisms are applied for the algorithms to aid on this matter. Also data pre-processing, such as feature transformations and normalization, have to be carried out for the classifier performance improvements. PCA approach enables to reduce the number of features by keeping only the principal components with the largest explained variance. However, this does not ensure the optimum separation of class boundaries. Effective ways to enhance 
the separability of classes (for example, types of holes in Fig. 4), usually must be found. Thus, care must be taken when selecting the signal features that are the most representative for every damage scenario. By combining signal information in time and frequency domains or finding other more important features, one can increase the distance between clusters of classes, improving separability.

\section{References}

[1] Wang L., Zhang Z.Automatic Detection of Wind Turbine Blade Surface Cracks Based on UAVtaken Images. IEEE Transaction on Industrial Electronics, vol. 64, 2017, pp. 7293-7303.

[2] Turkan Y., Hong J., Laflamme S. etc. Adaptive wavelet neural network for terrestrial laser scanner-based crack detection. Automation in Construction, vol. 94, 2018, pp. 191-202.

[3] Li P., Kong F., He Q. etc. Multiscale slope feature extraction for rotating machinery fault diagnosis using wavelet analysis. Measurement, vol. 46, 2013, pp. 497-505.

[4] Joshuva A., Sugumaran V. A data driven approach for condition monitoring of wind turbine blade using vibration signals through best-first tree algorithm and functional trees algorithm: A comparative study. ISA Transactions, vol.67, 2017, pp. 160-172.

[5] Jiménez A.A., Gómez Muñoz C.Q., García Máquez F. P. Machine Learning for Wind Turbine Blades Maintenance Management. Energies, vol. 11, 2018, pp. 13-28.

[6] Gaudenzi P., Nardi D., Chiappetta I., Atek S. etc. Sparse sensing detection of impact-induced delaminations in composite laminates. Composite Structures, vol. 133, 2015, pp. 1209-1219.

[7] Dhamande L.S., Chaudhari M.B. Compound gear-bearing fault feature extraction using statistical features based on time-frequency method. Measurement, vol. 125, 2018, pp. 63-77.

[8] Lu Y. S., Wang H.-W., Liu S.-H. An integrated accelerometer for dynamic motion systems. Measurement, vol. 125, 2018, pp. 471-475.

[9] Shen Z., Tan C.-Y., Yao K., Zheng L. etc. A miniaturized wireless accelerometer with micromachined piezoelectric sensing element. Sensors and Actuators A, vol. 241, 2016, .113-119.

[10] Bafroui H.H., Ohadi A. Application of wavelet energy and Shannon entropy for feature extraction in gearbox fault detection under varying speed conditions. Neurocomputing, vol. 133, 2014, pp. 437-445.

[11] Khan S., Yairi T. A review on the application of deep learning in system health management. Mechanical Systems and Signal Processing, vol. 107, 2018, pp. 241-265.

[12] Zhang G., Li C., Zhou H., Wagner T. Punching process monitoring using wavelet transform based feature extraction and semi-supervised clustering. Procedia Manufacturing, vol. 26, 2018, pp. 1204-1212.

[13] Stetco A., Dinmohammadi F., Zhao X., Robu V., et al. Machine learning methods for wind turbine condition monitoring: A review. Renewable Energy, vol. 133, 2019, pp. 620-635. 